\title{
Is DISH Painful?
}

Diffuse idiopathic skeletal hyperostosis (DISH) is a noninflammatory condition characterized by new bone growth affecting cancellous and cortical bone and particularly targeting the enthesis, that region where ligament, tendon, annulus fibrosis, and similar structures insert into cortical bone. Criteria for diagnosis are based on the radiological presence of entheseal ossification ${ }^{1,2}$, principally in the spine, where the new bone growth of DISH is ubiquitous.

DISH is a common condition with prevalence rates that vary depending on the criteria applied and the population studied. Estimates range from $3.8 \%$ of men and $2.6 \%$ of women in a Finnish population-based study ${ }^{3}$, to $22.4 \%$ of men and $13.4 \%$ of women in an Israeli hospital-based study $^{4}$. Its prevalence increases with age and hence with the aging population DISH will become more prevalent than before. There is a 2:1 male predominance in DISH, with severe radiological changes reported more commonly in males ${ }^{4}$. An association with obesity has been noted since the early description of the condition by Forestier and Rotes-Querol $^{5}$, and over time, type 2 diabetes and the metabolic syndrome have also been identified as comorbid conditions ${ }^{6,7}$. The metabolic factors associated with DISH, despite decades of inquiry, still likely relate to insulin-associated growth factors and the effect of those factors on bone and entheseal growth plates 8,9 .

As the population ages and as the proportion of metabolically vulnerable persons increases, so will the prevalence of DISH increase. Will this emerging epidemic of DISH increase the musculoskeletal health burden? In particular, will there be more pain and disability due to this disorder?

Symptoms traditionally attributed to DISH include restriction in movement of spinal joints and entheseal-related pain when peripheral exostoses caused by DISH are traumatized. Whether the condition itself is a cause of significant pain and whether it is a true disease entity are issues that continue to be debated ${ }^{10}$.

A number of studies have examined populations of patients with DISH compared to controls in order to better define symptoms that might relate to this disorder. Mata, et al compared clinical and laboratory features of 56 patients with DISH with 43 patients with lumbar spondylosis and 31 healthy controls free from major musculoskeletal disease ${ }^{11}$. Patient questionnaires addressed pain, stiffness, and psychological and physical disability, the latter assessed by the Health Assessment Questionnaire (HAQ) physical disability scale. A comprehensive physical examination was performed that included fibromyalgia tender point count, joint count, and range of motion at the shoulders, hips, and spine. The authors found that patients with DISH were more likely to report dysphagia (secondary to anterior cervical osteophytes) and previous episodes of entheseal pain, and to experience ongoing spinal pain and stiffness. Patients with DISH had a reduction in spinal mobility, but there was no difference in the number of fibromyalgia tender points, which were uncommon in all 3 patient groups. The HAQ revealed more disability in DISH patients than healthy controls, but this was not different from the level of disability experienced by patients with lumbar spondylosis.

Schlapbach, et al studied 106 patients with DISH and 178 control patients, all admitted to hospital for reasons other than back pain ${ }^{12}$. A rheumatologist blinded to diagnosis administered a structured questionnaire to each patient concerning spinal pain in the preceding 6 months. No difference was found between the 2 groups in terms of reported pain at any spinal level. There was no assessment of stiffness in the questionnaire, however, and a physical examination was not performed. Using a similar technique, the same group found that shoulder hyperostosis predisposed to shoulder pain ${ }^{13}$, while elbow hyperostosis led to no significant increase in elbow pain in hospitalized patients ${ }^{14}$.

Most of these studies used diagnostic criteria for DISH based on the presence of new bone formation in the thoracic or lumbar spine, with care to exclude patients with degenerative disc disease or with features that would fit with inflammatory spinal disease. In this setting it is unclear whether DISH is painful in its own right.

See Nonarticular tenderness and functional status in patients with DISH, page 1911

Personal non-commercial use only. The Journal of Rheumatology Copyright @ 2010 . All rights reserved. 
DISH, however, does not occur as a pure condition in the majority of persons. It can coexist with a number of other age-related degenerative diseases, including degenerative disc disease, degenerative joint disease, particularly spinal facet-joint degenerative disease, and peripheral osteoarthritis ${ }^{15}$.

In the spine the exuberant bone growth of DISH at entheseal areas and involving bone itself can add to established degenerative disease of discs and facet joints. For instance, facet-joint osteophytosis coupled with new bone growth from DISH occurring in annulus fibrosis entheseal regions and bony growth in anterior and posterior longitudinal ligaments plus the ligamentum flavum can all contribute to compromise of bony foramina designed to conduct neural structures from the spine to the periphery. Hence disorders such as spinal canal stenosis ${ }^{16}$, intervertebral foramina narrowing with neural impingement, and osteoarthritis of the facet joint may all be more problematic in patients with DISH compared to those without. Myelopathy and quadriplegia can also occur ${ }^{17,18}$. Dysphagia secondary to compression of the esophagus by large anterior cervical osteophytes is described in most series ${ }^{19}$.

In the periphery an association between DISH and degenerative hip disease has been reported dating back over 30 years $^{16}$. Associations have also been noted between DISH and other osteoarthritic regions such as the interphalangeal joints of the hand as well as the knee ${ }^{20}$, but better studies are required in this area. Additionally, as stated earlier, painful osteophytes in areas exposed to high mechanical trauma, such as the heel, are often associated with DISH. The metabolic correlates of DISH, particularly obesity, may compound the mechanical factors on involved weight-bearing structures.

In this issue of The Journal, Mader and colleagues ${ }^{21}$ examine other potential causes of pain and dysfunction in patients with DISH, namely the degree of nonarticular tenderness and its possible relationship to spinal hyperostosis. Using a comparator group of patients with osteoarthritis, they assessed fibromyalgia tender point counts as well as total tenderness score. DISH patients were found to have an increased total tenderness score, reflecting a lower pain threshold, and this score correlated with severity of thoracic bony bridges as graded on a scale of 0 to 4 . This suggests a relationship between DISH radiological changes and pain mechanisms. An increased number of tender points and total tenderness score both correlated with poor functional status, as measured by revised HAQ. This was independent of body mass index, which is itself known to be associated with poor functional status. The well recognized association of metabolic syndrome with DISH was confirmed in this population.

While these findings are novel, the study would be more robust if the control group was matched for body mass index and waist circumference, rather than "osteoarthritis." The effect of fitness and psychological distress on tenderness and function in DISH should also be determined. These factors will each influence tenderness and function in their own right $^{22}$. The patient group studied was also atypical in that $68.5 \%$ of DISH patients were female, while DISH is acknowledged to be a disease more common in males, and tenderness more common in females. In order to clarify these findings future studies will need to consider these biases.

Other studies have shown associations between spinal tightness and referred pain and tenderness ${ }^{23}$. The implication is that in persons with restrictions of movement in the spine, particularly the low cervical and low lumbar areas where normal biomechanical factors exaggerate stress on spinal structures, there are resultant referred pain phenome$\mathrm{na}^{24}$. Thus patients with DISH may have increased regional tenderness and tightness, and possibly pain, independent of the well described but infrequent neural complications of the disorder. Perhaps the patient with DISH is more vulnerable than others to comorbid degenerative spinal disease or the amplifying effects of unfitness or psychological distress on pain mechanisms. Based on the previous epidemiological studies and clinical observation it is currently difficult to know whether this possible mechanism has an important clinical effect.

The process of bone deposition and entheseal change in DISH is by itself asymptomatic. However, the overall effect of DISH acting on other musculoskeletal structures that are themselves undergoing degenerative change, such as discs and joints, can result in a variety of clinical consequences. In addition, the effect of DISH on spinal mechanical contributions to change in pain threshold and related pain mechanisms now needs to be considered.

These new potential DISH-associated mechanisms require further clarification, but once again focus interest on this most ancient and increasingly prevalent rheumatic disease.

GENE-SIEW NGIAN, MBBS, Rheumatology Fellow;

GEOFFREY O. LITTLEJOHN, MBBS (Hons), MD, MPH,

Director of Rheumatology,

Monash Medical Centre,

Associate Professor,

Monash University,

Melbourne, Australia

\section{REFERENCES}

1. Resnick D, Niwayama G. Radiographic and pathologic features of spinal involvement in diffuse idiopathic skeletal hyperostosis (DISH). Radiology 1976;119:559-68.

2. Utsinger PD. Diffuse idiopathic skeletal hyperostosis. Clin Rheum Dis 1985;11:325-51.

3. Julkunen H, Heinonen OP, Knekt P, Maatela J. The epidemiology of hyperostosis of the spine together with its symptoms and related mortality in a general population. Scand J Rheumatol 1975;4:23-7.

4. Bloom RA. The prevalence of ankylosing hyperostosis in a Jerusalem population - with description of a method of grading the extent of the disease. Scand J Rheumatol 1984;13:181-9. 
5. Forestier J, Rotes-Querol J. Senile ankylosing hyperostosis of the spine. Ann Rheum Dis 1950;9:321-30.

6. Littlejohn GO. Insulin and new bone formation in diffuse idiopathic skeletal hyperostosis. Clin Rheumatol 1985;4:294-300.

7. Mader R, Sarzi-Puttini P, Atzeni F, Olivieri I, Pappone N, Verlaan $\mathrm{JJ}$, et al. Extraspinal manifestations of diffuse idiopathic skeletal hyperostosis. Rheumatology 2009;48:1478-81.

8. Littlejohn GO, Smythe HA. Marked hyperinsulinemia after glucose challenge in patients with diffuse idiopathic skeletal hyperostosis. J Rheumatol 1981;8:965-8.

9. Denko CW, Malemud CJ. Body mass index and blood glucose: correlations with serum insulin, growth hormone, and insulin-like growth factor-1 levels in patients with diffuse idiopathic skeletal hyperostosis (DISH). Rheumatol Int 2006;262:292-7.

10. Hutton C. DISH ... a state not a disease? Br J Rheumatol 1989;28:277-8

11. Mata S, Fortin PR, Fitzcharles MA, Starr MR, Joseph L, Watts CS, et al. A controlled study of diffuse idiopathic skeletal hyperostosis. Clinical features and functional status. Medicine Baltimore 1997;76:104-17.

12. Schlapbach P, Beyeler C, Gerber NJ, van der Linden S, Burgi U, Fuchs WA, et al. Diffuse idiopathic skeletal hyperostosis (DISH) of the spine: a cause of back pain? A controlled study. Br J Rheumatol 1989;28:299-303.

13. Beyeler C, Schlapbach P, Gerber NJ, Sturzenegger J, Fahrer H, van der Linden S, et al. Diffuse idiopathic skeletal hyperostosis (DISH) of the shoulder: a cause of shoulder pain? Br J Rheumatol 1990;29:349-53.

14. Beyeler C, Schlapbach P, Gerber NJ, Fahrer H, Hasler F, van der Linden SM, et al. Diffuse idiopathic skeletal hyperostosis (DISH) of the elbow: a cause of elbow pain? A controlled study. Br J Rheumatol 1992;31:319-23.
15. Mader R, Dubenski N, Lavi I. Morbidity and mortality of hospitalized patients with diffuse idiopathic skeletal hyperostosis. Rheumatol Int 2005;26:132-6.

16. Arlet J, Abiteboul M, Mazieres B, Adam P, Roulleau J. Acquired stenosis of the lumbar canal and vertebral hyperostosis [French]. Rev Rhum Mal Osteoartic 1983;50:635-41.

17. Stechison MT, Tator CH. Cervical myelopathy in diffuse idiopathic skeletal hyperostosis. Case report. J Neurosurg 1990;73:279-82.

18. Pascal-Moussellard H, Drossard G, Cursolles JC, Catonne Y, Smadja D. Myelopathy by lesions of the craniocervical junction in a patient with forestier disease. Spine Phila Pa 1976 2006; 31:E557-60.

19. Eviatar E, Harell M. Diffuse idiopathic skeletal hyperostosis with dysphagia [review]. J Laryngol Otol 1987;101:627-32.

20. Smythe HA. The mechanical pathogenesis of generalised osteoarthritis. J Rheumatol 1983;10 Suppl 9:11-2.

21. Mader R, Novofastovski I, Rosner E, Adawi M, Herer P, Buskila D. Nonarticular tenderness and functional status in patients with diffuse idiopathic skeletal hyperostosis. J Rheumatol 2010; 37:1911-6.

22. Neumann L, Lerner E, Glazer Y, Bolotin A, Shefer A, Buskila D. A cross-sectional study of the relationship between body mass index and clinical characteristics, tenderness measures, quality of life, and physical functioning in fibromyalgia patients. Clin Rheumatol 2008;27:1543-7.

23. Littlejohn G, Granges G. The relationship between vertebral dysfunction and clinical features of the fibromyalgia syndrome. J Orthop Rheumatol 1995;8:97-105.

24. Smythe H. Referred pain and tender points. Am J Med 1986; $81: 90-2$.

J Rheumatol 2010;37:1797-9; doi:10.3899/jrheum.100519 